\title{
Oral plasmablastic lymphoma as the first manifestation of AIDS*
}

\author{
Daniela Assis do Vale ${ }^{1}$ \\ Danielle Lima Corrêa de Carvalho ${ }^{1}$ \\ Karem López Ortega ${ }^{3}$
}

\author{
Carolina Martelli Rogado² \\ Marilia Trierveiler ${ }^{3}$
}

DOI: http:/ / dx.doi.org/10.1590/abd1806-4841.20175417

\begin{abstract}
Plasmablastic lymphoma is a non-Hodgkin lymphoma characterized by its plasmacytic differentiation and predilection for the oral cavity. It is among the lymphomas most commonly associated with AIDS. This report details a case of a HIV-positive patient with a 1-month history of an exophytic mass in the gingival area of the upper left quadrant. The diagnosis of plasmablastic lymphoma was made based on its histopathological and immunophenotypical features. She was treated with chemotherapy followed by autologous hematopoietic stem cell transplantation. Despite complete resolution of the lesion, the patient died of cardiorespiratory arrest. This case illustrates plasmablastic lymphoma as the first clinical manifestation of AIDS, highlighting the importance of differentiating between a potentially malignant lesion and other pathologic processes.
\end{abstract}

Keywords: Acquired Immunodeficiency Syndrome; HIV; Lymphoma, AIDS-Related

\section{INTRODUCTION}

Plasmablastic lymphoma (PBL) is a non-Hodgkin lymphoma, variant of the diffuse large B-cell lymphoma, rare, aggressive and characterized by its plasmacytic differentiation. PBL occurs in patients with some form of immunosuppression, especially the one caused by HIV (human immunodeficiency virus) infection, and $80 \%$ of the cases associated with HIV are also positive for Epstein-Barr virus (EBV). ${ }^{1-4}$

It affects more men, with a 4:1 ratio and mean age of 39 years. However, due to its rarity, the exact incidence remains unknown. ${ }^{1,2,4}$

The clinical presentation is of an exophytic, painless, rapidly growing and sometimes ulcerated lesion. It has predilection for the mouth, particularly the gingivae and palate. Submandibular and cervical lymphadenopathy, as well as B-cell lymphoma symptoms like fever, night sweats and weight loss can be present. ${ }^{1,5,6}$

The differential diagnosis of an expanding oral lesion includes from infectious dental processes to malignancies. Malig- nancies include squamous cell carcinoma, metastatic tumors and Kaposi sarcoma. Besides plasmablastic lymphoma, other types of lymphoma can also occur in the oral cavity, including diffuse large B-cell lymphoma, plasmacytomas and Burkitt lymphoma. ${ }^{5}$

There is no standard treatment for plasmablastic lymphoma. However, chemotherapy remains as the treatment of choice. Recently, autologous hematopoietic stem cell transplantation has been shown to be an option, increasing patient survival. ${ }^{1,2,5}$

\section{CASE REPORT}

Fifty-six-year-old female Caucasian pacient, smoker, was referred for the diagnosis of a lesion on the gingiva.

When the lesion first appeared, 1 month one month before the appointement, the patient sought a dental surgeon that upon noticing intense bone loss on the right posterior maxillary region on the radiograph, suggestive of periodontal destruction, opted to

Study submitted on 22.11.2015

Approved by the Advisory Board and accepted for publication on 16.04.2016

* Study conducted at Special Patient Unit and Oral and Maxillofacial Pathology of the Department of Oral Medicine of the Faculdade de Odontologia Universidade de São Paulo (FO-USP) - São Paulo (SP), Brazil.

Financial Support: None.

Conflict of Interests: None.

Post-graduation Program in Dental Sciences -Area of Oral and Maxillofacial Pathology and Special Patients of the Faculdade de Odontologia - Universidade de São Paulo (FO-USP) - São Paulo (SP), Brazil.

Special Care Dentistry Center, Faculdade de Odontologia - Universidade de São Paulo (CAPE-FOUSP), São Paulo (SP), Brazil.

Department of Oral Medicine, discipline of Oral and Maxillofacial Pathology of the Faculdade de Odontologia - Universidade de São Paulo (FO-USP) - São Paulo (SP), Brazil.

C2017 by Anais Brasileiros de Dermatologia 
remove the affected teeth and prescribe antibiotics. Since there was no improvement, the patient was referred to our clinic.

Concomitantly, the patient was under investigation of erythematous patches, spread throughout her body that appeared 11 months earlier, leading to the diagnosis of HIV infection and cutaneous rash. She did not have any other associated conditions or history of opportunistic infections.

CD4+ cell count and viral load (VL) before antiretroviral treatment was 146 cells $/ \mathrm{mm}^{3}$ and 1,111,978 copies/ml, respectively. Fifteen days after starting treatment with lamivudine, tenofovir and efavirenz, CD4+ cells went to 189 cells $/ \mathrm{mm}^{3}$ and VL to 13,504 copies/ml

We collected a detailed history and on physical examination a painless tumor with an erythematous surface and an ulcerated area was observed on the right upper gingiva (Figure 1). The radiograph showed intense, generalized periodontal bone loss. Incisional biopsy was performed, and the sample was sent to the laboratory for analysis.

Histopathology showed a fragment of lymphoid neoplasia, represented by a layer of bulky cells, similar to immunoblasts. Intense pleomorphism and multiple mitotic figures were also ob- served (Figure 2A). Immunohistochemistry revealed positivity to anti-plasma cell marker and immunoglobulin kappa light chain, and was negative to anti-CD3, CD20 and lambda light chain, confirming monoclonality and plasmacytic origin of the tumor. In situ hybridization was positive for EBV (Figure 2B). The final diagnosis of PBL was made based on the histological and immunophenotypic features.

The patient was referred to chemotherapy treatment, having had 9 cycles of EPOCH (etoposide, prednisone, vincristine, cyclophosphamide and doxorubicin). Afterwards, she presented with complete remission of the lesion (Figure 3). She then underwent autologous hematopoietic stem cell transplantation and progressed with sepsis, bronchospasm and died due to cardiorespiratory arrest 10 months after being diagnosed with PBL.

\section{DISCUSSION}

With the advent of HAART, the incidence of opportunistic infections as well as the morbidity and mortality associated to HIV infection decreased, a trend that could also be seen with oral lesions. ${ }^{7,8}$ The incidence of lymphomas associated with HIV infection in general decreased $50 \%$ after HAART was made avail-
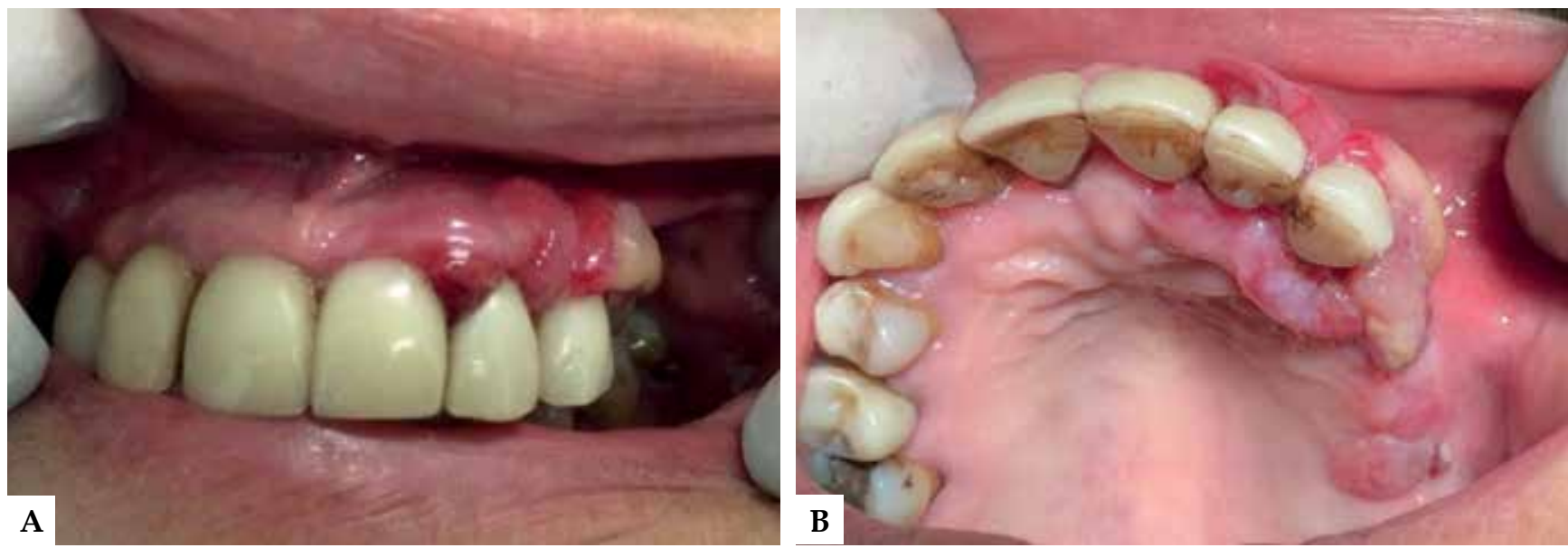

Figure 1: A. Clinical aspect of the lesion on the first visit. B. Clinical aspect of the lesion on the first visit
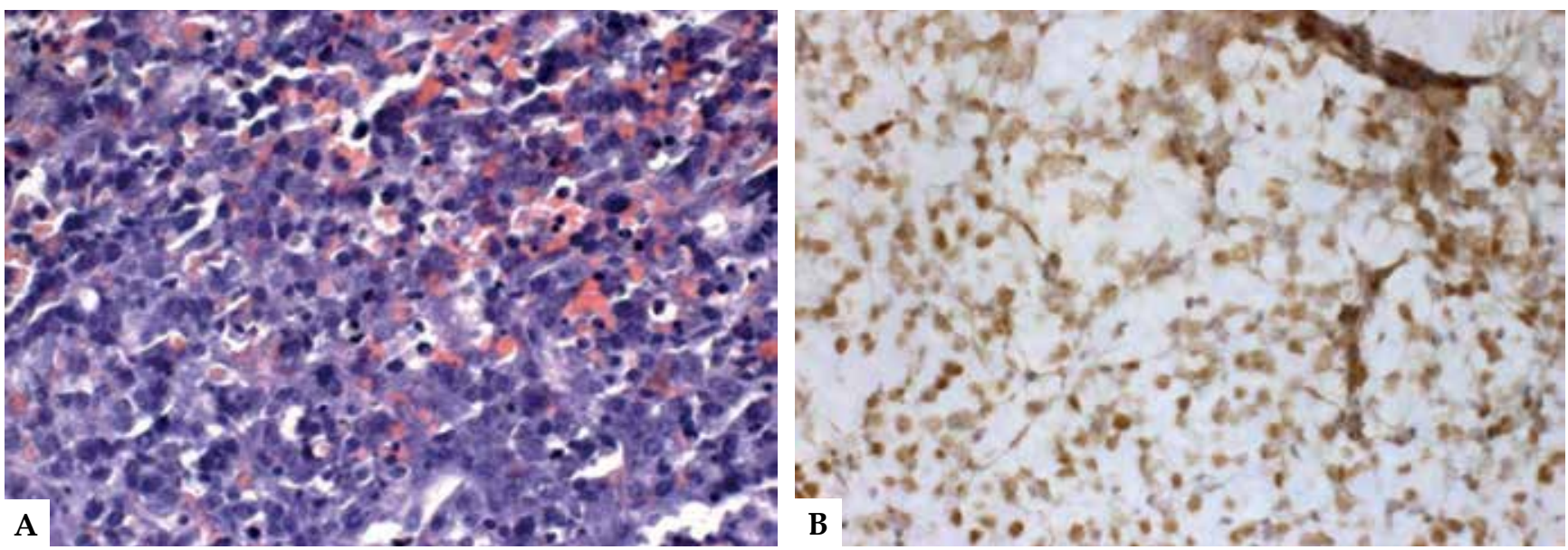

Figure 2: A. Histopathology showing lymphoid proliferation and intense cellular pleomorphism (Hematoxylin \& eosin, X40). B.

hybridization confirming the presence of Epstein-Barr virus $(X 40)$ 

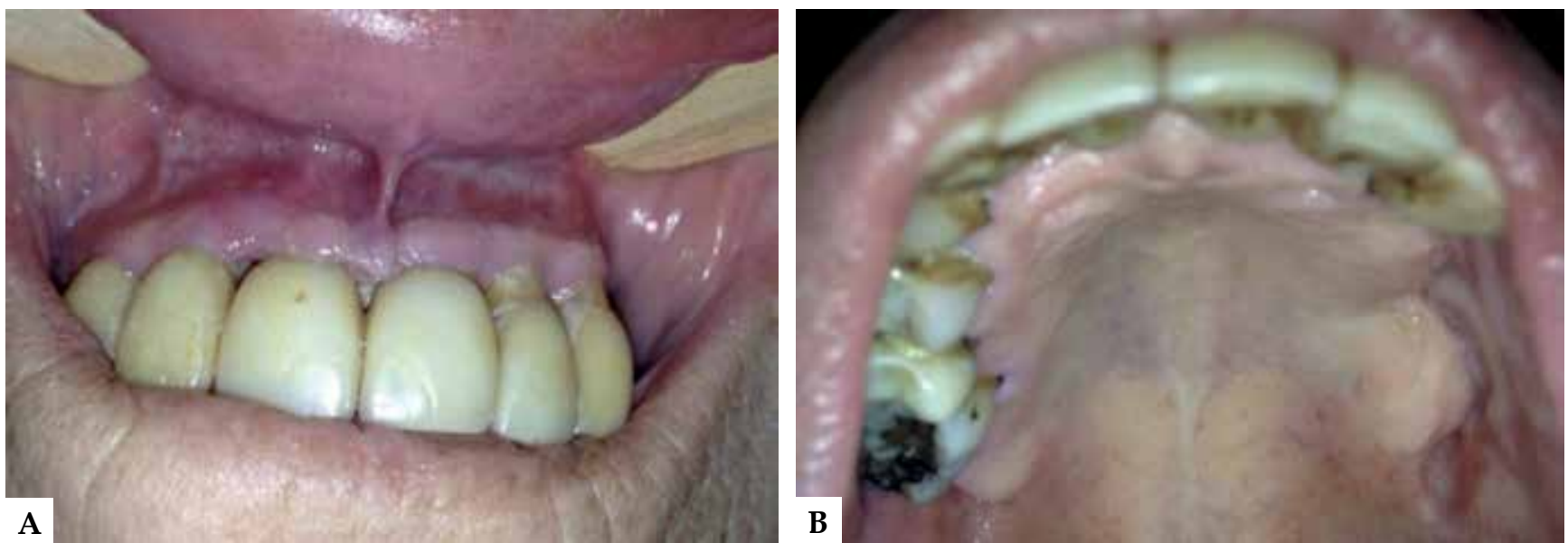

Figure 3: A AND B. Complete remission of the lesion after chemotherapy

able. However, because of the small number of cases, there is not enough information to affirm that the advent of HAART altered the prevalence of PBL. ${ }^{7}$

The diagnosis of oral lymphomas can be challenging because there is low clinical suspicion, leading to diagnostic errors and delayed treatment. ${ }^{9}$ A careful assessment, knowledge about HIV infection and its oral manifestations will help correctly and promptly diagnosing malignant lesions, avoiding situations such as in our case, where the initial diagnosis was of a dental infection.
Even though the prognosis of PBL remains somber, with mean survival of 11 to 13 months, the combination of HAART with chemotherapy has yielded better survival for HIV-positive patients. Promising results have also been reported with the use of targeted therapies against EBV, such as zidovudine, ganciclovir and interleukin-2., ${ }^{40}$

PBL, an AIDS defining lesion, can be the first clinical manifestation of HIV infection and physicians must be vigilant to make an accurate and early clinical diagnosis, being able to differentiate a potentially malignant lesion from other neoplastic or infectious processes in patients, regardless of their serology, increasing their chances of survival.]

\section{REFERENCES}

1. Castillo JJ, Reagan JL. Plasmablastic lymphoma: a systematic review. ScientificWorldJournal. 2011;11:687-96.

2. Al-Malki MM, Castillo JJ, Sloan JM, Re A. Hematopoietic cell transplantation for plasmablastic lymphoma: a review. Biol Blood Marrow Transplant. 2014;20:187784.

3. Corti M, Minué G, Campitelli A, Narbaitz M, Gilardi L. An Aggressive Plasmablastic Lymphoma of the Oral Cavity as Primary Manifestation of Acquired Immunodeficiency Syndrome: Case Report and Literature Review. Int Arch Otorhinolaryngol. 2015;19:354-8

4. Elyamany G, Al Mussaed E, Alzahrani AM. Plasmablastic Lymphoma: A Review of Current Knowledge and Future Directions. Adv Hematol. 2015;2015:315289.

5. Francischini E, Martins FM, Braz-Silva PH, Magalhães MH, Ortega KL. HIVassociated oral plasmablastic lymphoma and role of adherence to highly active antiretroviral therapy. Int J STD AIDS. 2010;21:68-70.

6. Bibas M, Castillo JJ. Current knowledge on HIV-associated Plasmablastic Lymphoma. Mediterr J Hematol Infect Dis. 2014;6:e2014064.

7. Medel N, Hamao-Sakamoto A. A case of oral plasmablastic lymphoma and review of current trends in oral manifestations associated with human immunodeficiency virus infection. J Oral Maxillofac Surg. 2014;72:1729-35.
8. Ortega KL, Vale DA, Magalhães MH. Impact of PI and NNRTI HAART-based therapy on oral lesions of Brazilian HIV-infected patients. J Oral Pathol Med. 2009;38:489-94.

9. Sirsath NT, Lakshmaiah KC, Das U, Lokanatha D, Chennagiri SP, Ramarao C. Primary extranodal non-Hodgkin's lymphoma of oral cavity--a single centre retrospective study. J Cancer Res Ther. 2014;10:945-50.

10. Castillo J, Pantanowitz L, Dezube BJ. HIV-associated plasmablastic lymphoma: lessons learned from 112 published cases. Am J Hematol. 2008 0ct;83:804-9.

\footnotetext{
ENDEREÇO PARA CORRESPONDÊNCIA:

Prof. Dra. Karem López Ortega

Faculdade de Odontologia - Universidade de São Paulo

Av. Prof. Lineu Prestes, 2227 - Cidade Universitária

05508-000 - São Paulo -SP - Brazil

E-mail:klortega@usp.br
}

How to cite this article: Vale DA, Rogado CM, Caravalho DLC, Trierveiler M, Ortega KL. Oral plasmablastic lymphoma as the first manifestation of AIDS. An Bras Dermatol. 2017;92(5 Suppl 1): 110-2. 